\title{
Research on the Effect of Carbonate Reservoir Pore Structure on Oil Displacement Efficiency
}

\author{
Ma Wenguo ${ }^{1}$ and Gong $\mathrm{He}^{2}$ \\ ${ }^{1}$ Key Laboratory of Enhancing Oil and Gas Recovery of Education Ministry, \\ Northeast Petroleum University, Daqing, 163318, China \\ ${ }^{2}$ Northeast Petroleum University, China \\ ${ }^{1}$ mawenguo110@126.com, ${ }^{2}$ gonghe0321@163.com
}

\begin{abstract}
Characteristics of pore structure have an important influence on the water flooding development. In order to improve the recovery rate, it is important to have a research on the relationship between pore structure and oil displacement efficiency. The permeability

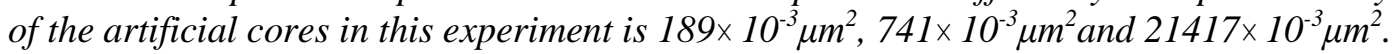
We used the CT technology method to scan the pore structure of the three cores, and did oil displacement experiment to research the effect of pore structure on the oil displacement efficiency. The result shows that the pore and throat common affect oil displacement efficiency: the bigger pore and throat radius is, the better effect of water flooding and polymer flooding is; the smaller pore and throat radius is, the worse effect of water flooding and polymer flooding is. The experiment has studied the influence of pore structure on oil displacement efficiency deep into microcosmic without damaging the core skeleton, improved the basis of oil recovery from the micro and the mechanism.
\end{abstract}

Keywords: Pore Structure, Oil Displacement Efficiency, the Effect of Polymer Flooding, Enhanced Oil Recovery

\section{Introduction}

The petroleum is non renewable resources. With the increase of the world oil consumption, new oil geological reserves become more and more difficult, and the exploitation of oil quality is becoming worse and worse. To enhance the oil recovery has become a major problem of the research of oil exploitation. Oil exist in reservoir space that formed by different types of pores, so the microscopic pore structure of the reservoir plays an important part on the recovery rate. The previous research on the influence factors of oil displacement efficiency, most are doing more work from the geological conditions and development conditions. For the study of the geological conditions are often limited to the macro level. Therefore, for the block of similar geological conditions and development conditions, the reason of different oil displacement can not explain clearly. The limitations of the research brings difficulties to the study on mechanism and the prediction of polymer flooding, and also impact the scientific establishment for the polymer flooding development planning.

Characteristics of pore structure are the main factor to influence reservoir capability and oil and gas resources. Therefore, the pore structure characteristics of rock clearly play a key to improve the productivity of oil and gas reservoir and oil and gas recovery. The description method of the rock pore structure characteristics include: laboratory experiments and field well logging evaluation. The indoor experiment method is the main method, at present, also the most widely used to describe and evaluate the rock pore structure, mainly includes: the capillary pressure curve method (the high pressure semi permeable membrane, mercury intrusion and centrifuge method etc.), thin section method, 
scanning electron microscope and CT scanning method. CT scanning method is the establishment of micro pore structure of the most direct and accurate, with the rapid development of CT technology, high spatial resolution and accuracy of micro pore structure of the support. The research carry out the study on the relationship between the oil displacement effect from the reservoir microscopic pore structure and polymer deep into micro level, reveals the inner link of porous media and oil displacement effect from the micro level.

\section{Basic Principles of CT Scanning}

An X-ray (radiography) system produces two-dimensional shadow images of complete internal three-dimensional structures, but in a single two-dimensional shadow projection the depth information is completely mixed. Only an X-ray tomography system allows us to visualize and measure complete three-dimensional object structures without sample preparation or chemical fixation. Typically, the spatial resolution of conventional medical CT-scanners is in the range of $1 \sim 2.5 \mathrm{~mm}$, which corresponds to $1 \sim 10$ cubic mm voxel (volume element) size. Computerized X-ray microscopy and micro tomography now gives possibilities to improve the spatial resolution by seven to eight orders in the volume terms.

Any X-ray shadow image is corresponding to a two-dimensional projection from the three-dimensional object. Each point on the shadow image contains the integration of absorption information inside the three-dimensional object in the corresponding partial X-ray beam.

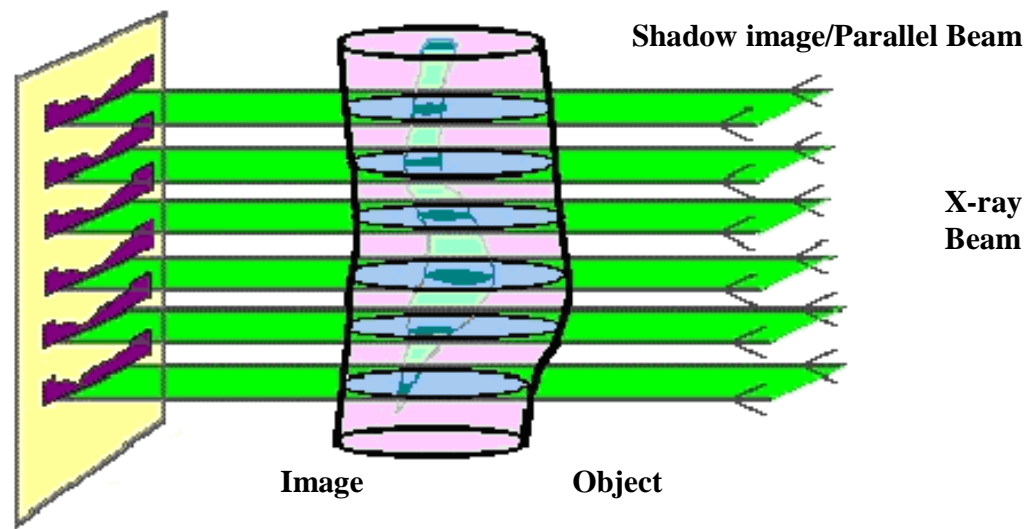

Figure 1. Parallel Beam Geometry

However most X-ray sources are not able to generate parallel beams. In a real case, one will use a point source which produces a cone X-ray beam in the object area. For tomography reconstruction the solution of this problem can be found by the reordering of the shadow information. In fan beam geometry the reconstructed slices will show some distortions far from the optical axis. In order to solve these errors we have to use a 3D cone beam reconstruction algorithm (such as Feldkamp) in order to take into account the thickness of the object. In other words the rays going through the front and the back of the object will not be projected on the same row of the detector. This is a non-linear operation which results in the fact that any noise in the small signal areas can produce significant errors in reconstruction. To eliminate these errors an averaging of initial data can be used. On the other hand we can try to improve the signal to noise ratio in the shadow image by optimizing the exposure time in order to reach the most representative information. 


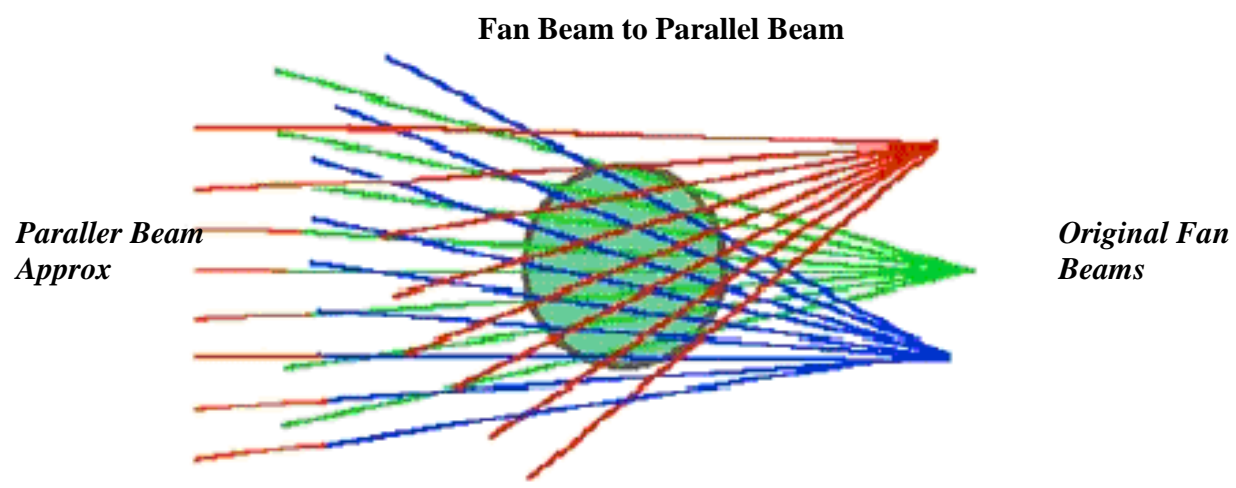

\section{Figure 2. Reordering the X-Rays from Fan Beam to Parallel Beam}

After reconstructing the raw data cross section, for 8 bit images 256 gray scales are used. Therefore, we have to convert the 16 bit information from the floating point matrix into a gray scale image. The minimum and maximum values are selected. All values between these will be displayed as half tone. In a normal image, all attenuation values below the minimum will be white while everything above the maximum will be displayed as black. The reconstructed array will be shown as a half-tone image of the cross section with linear conversion to 256-grades of gray inside the selected density interval.

The following image summarizes all action and steps to generate the cross sectional data:

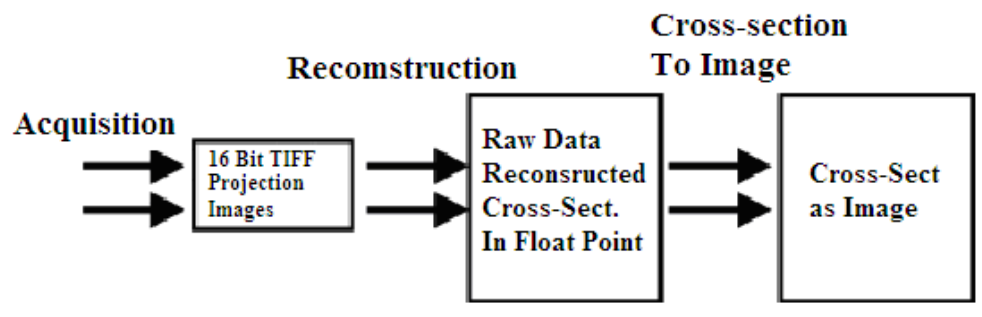

Figure 3. From Acquisition to the final Reconstructed Cross Section Image

\section{Mechanism of Polymer Flooding}

Compared with other chemical flooding, polymer flooding is relatively simple mechanism, namely the polymer can improve water/oil mobility ratio by increasing the viscosity of the injected water and reducing water permeability reservoir, adjust the injection profile, expand swept volume, enhance oil recovery.

Polymer flooding can improve the driving pressure difference inside the rock; the injected liquid can overcome capillary resistance in small pore and go into small pore to oil displacement. Its role is mainly manifested in three aspects: Firstly, the adsorption: As a large number of polymer adsorbed on the pore walls, which reduce the flow capacity of the water phase, the oil phase is not much impact on, in the same oil saturation, relative permeability of the oil phase increased compared water flooding. Secondly, the viscous effects: Since the viscoelastic polymer reinforce viscous effect of irreducible oil of water, the irreducible oil flow again and entrain out with the polymer solution. Thirdly, increasing the driving pressure difference: polymer improves the driving pressure inside the rock, making the injected liquid can overcome big capillary resistance in small holes and flood into the tiny pores. 
In general, the mechanism of polymer flooding mainly display in: (1) Improving sweep efficiency of macro $\left(E_{V}\right)$. After the polymer into the formation, it will increase the viscosity of the injected water, reduce water permeability, adjust the oil injection profile, improve the plane heterogeneity, increase the thickness of washing, expand the swept volume of the aqueous phase and improve macroscopic sweep efficiency. (2) Increasing the microscopic oil displacement efficiency $\left(E_{D}\right)$. Selecting the appropriate reservoir, correcting injection system design, polymer flooding can improve the recovery of $10 \%$. Domestic and foreign experts believe that this is due to the polymer has viscoelastic effects at a certain injection speed so as to improve the oil displacement efficiency. Its main mechanism of displacement:(1)viscoelastic polymer solution drag and carry irreducible oil on the blind side of the pore.(2) polymer solution on continuous film carrying mechanism.(3)viscoelastic polymer solution on irreducible oil of pore throats carrying mechanism.(4)viscoelastic polymer solution on irreducible oil of traps carrying mechanism

\section{Experiment}

\subsection{CT Scanning Experiment}

The experiment use high frequency CT machine SkyScan1172 and the corresponding software: Data Viewer, CTAN, ANT and CTVol and so on, rock cutting machine, artificial cores with permeability of $189 \times 10^{-3} \mu \mathrm{m}^{2}, 741 \times 10^{-3} \mu \mathrm{m}^{2}$, and $21417 \times 10^{-3} \mu \mathrm{m}^{2}$.

Experimental procedure:

(1) Use machine cut the core into the size of CT holder requirements;

(2) The experimental instrument is preheated for 15 minutes;

(3) Through the observation of the sample position, adjust to the appropriate position to start scanning;

(4) Reconstruct the obtained data images into 2D cross section via the Nrecon;

(5) Analyze and calculate of the parameters of pore structure by using CTAN software;

(6) Select the 30 50 layer for 3D reconstruction. Select appropriate gray value range. Reconstruct rock skeleton. Select inverse gray range and reconstruct rock pore;

(7) The experimental data analysis and summary.

\subsection{Water Flooding Experiment}

The experiment saturated the cores with $6.778 \mathrm{~g} / \mathrm{L}$ simulated water, and use $3.7 \mathrm{~g} / \mathrm{L}$ simulated water in water flooding. The experimental oil is made up of the oil for Daqing oil factory dehydration of crude oil and aviation kerosene. The viscosity of simulation oil is $9.8 \mathrm{cp}$. The experiment is at a constant temperature of $45^{\circ} \mathrm{C}$ in laboratory.

Experimental procedure:

(1) Check the ring pressure and make it keep 4Mpa.

(2) ISCO pump is connected to middle container with simulation water, and exhausting. After they are connected to the core holder end of the valve, open the valve, and connect six-way pressure gauge to the other end of the core holder and open the valve on the six-gauge to exhaust.

(3) U-shaped tube is connected with the core holder at the other end to the valve, opening the valve, ISCO pump flow is set to $0.1 \mathrm{ml} / \mathrm{min}$ injection.

(4) Measure pressure, fluid withdrawal rate, oil production once every $10 \mathrm{~min}$.

(5) Using the above data to calculate the cumulative oil production, recovery ratio and water ratio, when the water content is $98 \%$, stopping water displacing. 


\subsection{Polymer Flooding Experiment}

Polymer solution was configured with $0.508 \mathrm{~g} / \mathrm{L}$ simulated water. Polymer solution used for the experiment is at the concentration of $1.0 \mathrm{~g} / \mathrm{L}$, molecular weight of 25 million.

Experimental procedure:

(1) After water displacing, close the six valve and intermediate container of water, open the connection valve and connect polymer intermediate container, begin polymer flooding inject, $0.1 \mathrm{ml} / \mathrm{min}$, injection of $0.57 \mathrm{PV}$.

(2) Measure pressure, fluid withdrawal rate, oil production once every 10min.

(3) Using the above data to calculate the cumulative oil production, recovery ratio and water ratio, when the polymer injection amount is $0.57 \mathrm{PV}$, begin subsequent water flooding, when the water content is $98 \%$, stopping water displacing.

\section{Result and Analysis}

The experiment gets the distribution of pore radius, throat radius and pore-throat ratio of different permeability artificial core.

Figure 4 shows the histogram of pore radius distribution frequency: the cores permeability of $189 \times 10^{-3} \mu \mathrm{m}^{2}$ and $741 \times 10^{-3} \mu \mathrm{m}^{2}$ have the pore radius distribution frequency mainly concentrated in $5.3 \mu \mathrm{m} \sim 7.5 \mu \mathrm{m}$ range; The pore radius of the core permeability of $21417 \times 10^{-3} \mu \mathrm{m}^{2}$ is still distributed part in the low value, but overall, the majority of pore radius is mainly distributed in the high range.

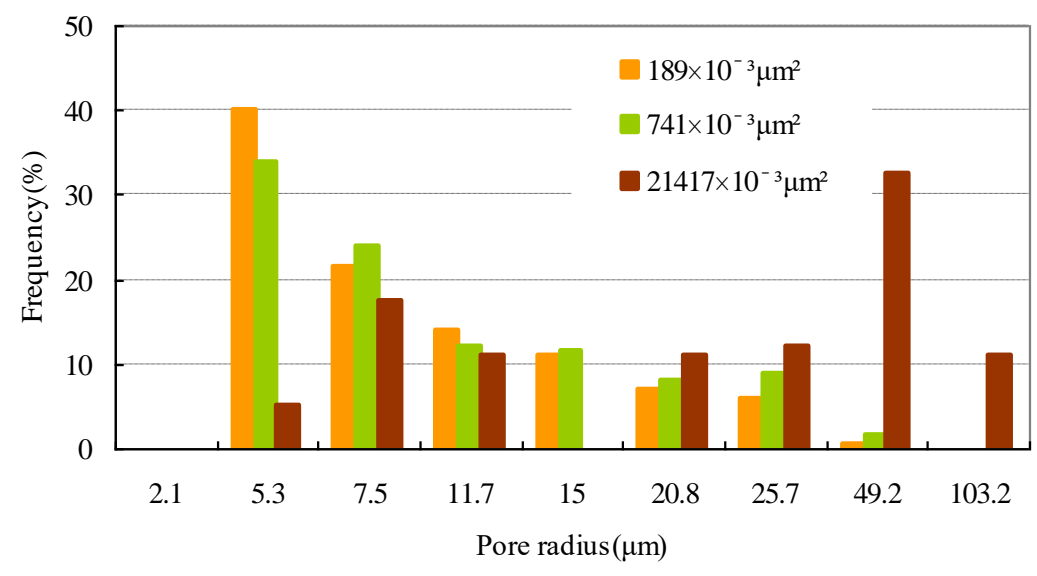

Figure 4. Distribution Frequency of Pore Radius

Figure 5 shows the histogram of throat radius distribution frequency: the cores permeability of $189 \times 10^{-3} \mu \mathrm{m}^{2}$ and $741 \times 10^{-3} \mu \mathrm{m}^{2}$ have the throat radius distribution frequency mainly concentrated in $1.0 \mu \mathrm{m} \sim 2.5 \mu \mathrm{m}$ range; the throat radius of the core permeability of $21417 \times 10^{-3} \mu \mathrm{m}^{2}$ mostly located in the high range. 


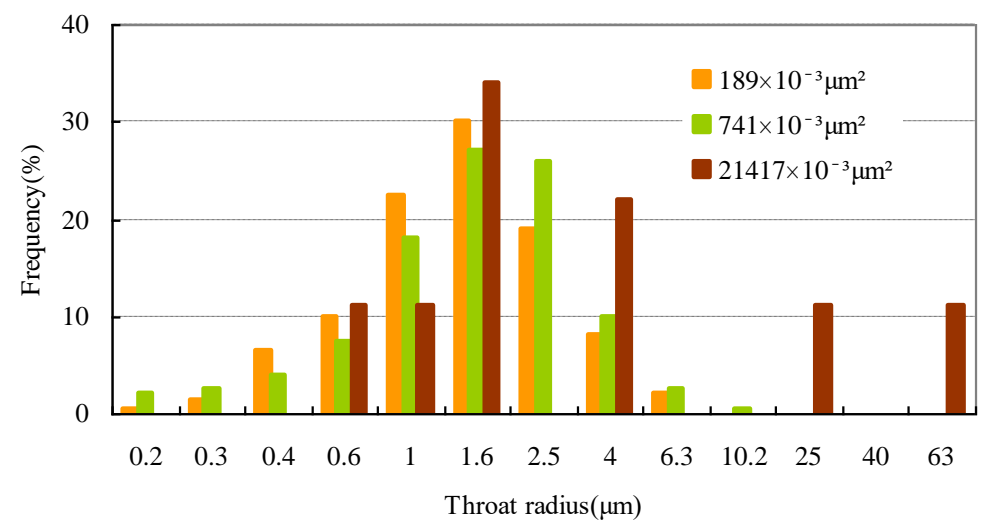

Figure 5. Distribution Frequency of Throat Radius

Figure 6 shows the histogram of pore-throat ratio distribution frequency: the distribution range of pore-throat ratio of the core permeability of $189 \times 10^{-3} \mu \mathrm{m}^{2}$ is wide, $80.5 \%$ of the pore-throat ratio is greater than 3 ; the distribution range of pore-throat ratio of the core permeability of $741 \times 10^{-3} \mu \mathrm{m}^{2}$ is wide, $79.2 \%$ of the pore-throat ratio is greater than 3 ; it can be seen from the graph, the pore-throat ratio distribution frequency of the core permeability of $21417 \times 10^{-3} \mathrm{\mu m}^{2}$ is single, the distribution frequency at 3 is more, up to $22.2 \%, 77.8 \%$ the pore-throat ratio is greater than 3 .

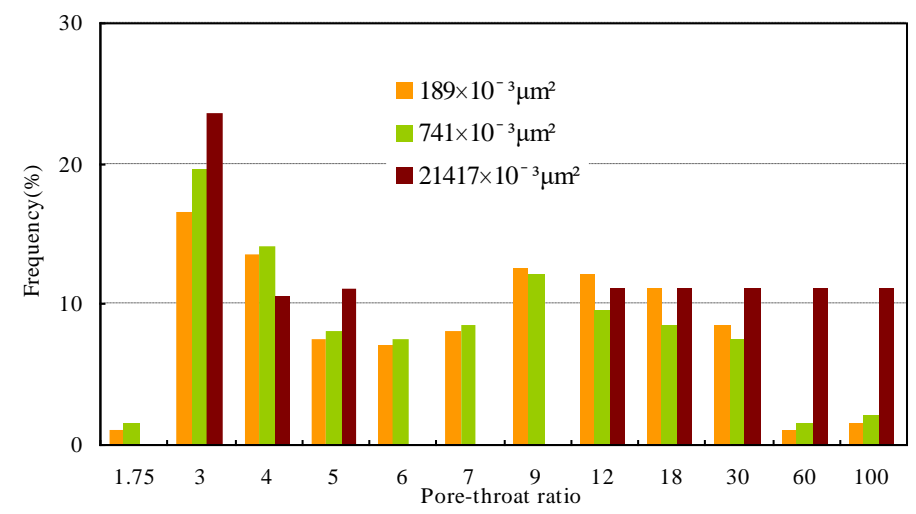

Figure 6. Distribution Frequency of Pore-Throat Ratio

After the experiments of vacuuming, water saturating, oil saturating, water oil displacement experiment was done on the three cores of different permeability. We measured pressure, fluid withdrawal rate, oil production once every 10min during water flooding stage and use the above data to calculate the cumulative oil production, recovery ratio and water ratio. When the water content is $98 \%$, stop water flooding experiment and begin polymer flooding experiment(the molecular weight of 25 million, the concentration of $1.0 \mathrm{~g} / \mathrm{L}$ ), injection of $0.57 \mathrm{PV}$.

The recovery rate of water flooding and polymer flooding stage are as shown in the Figure 6.Water flooding stage: the recovery rate of the $189 \times 10^{-3} \mu \mathrm{m}^{2}$ permeability core is $36.1 \%$; the recovery rate of the $741 \times 10^{-3} \mu \mathrm{m}^{2}$ permeability core is $37.4 \%$; the recovery rate of the $21417 \times 10^{-3} \mu \mathrm{m}^{2}$ permeability core is $64.2 \%$.

Recent studies show that after the viscoelastic fluid and viscous fluid displace core in the same condition, the residual oil saturation of the former is significantly lower than that of the latter. This shows that, viscoelastic polymer solution can not only improve the macroscopic sweep efficiency, but also can greatly improve the microscopic oil 
displacement efficiency. In the experiment, we determined the viscosity of the polymer solution, and the result of determination is shown in Figure 6. Figure 7 shows the experimental modulus curves of polymer solution. $G^{\prime}$ is called storage modulus and $G^{\prime \prime}$ is called loss modulus. For the viscous properties of polymer solution, part of the energy is dissipated or loss; and for the elastic properties, part of the energy is stored. The storage modulus $\left(G^{\prime}\right)$ reflects the elasticity of viscoelastic fluid, and the loss modulus $\left(G^{\prime \prime}\right)$ reflects the stickiness of viscoelastic fluid.

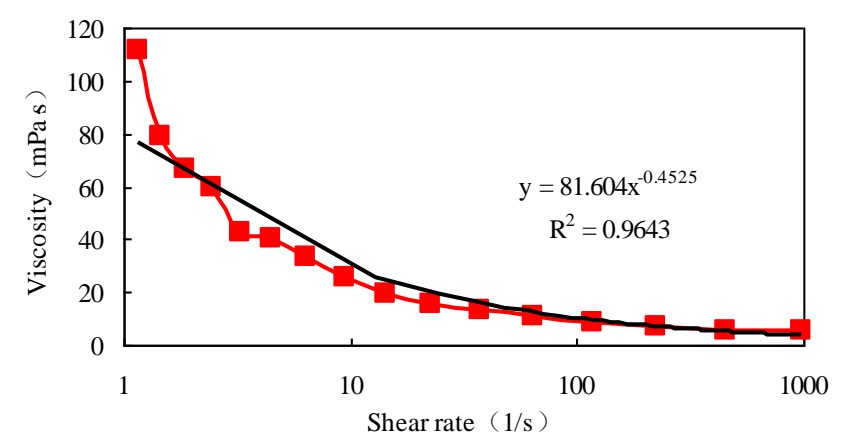

Figure 7. Viscosity Curve of the Polymer Solution

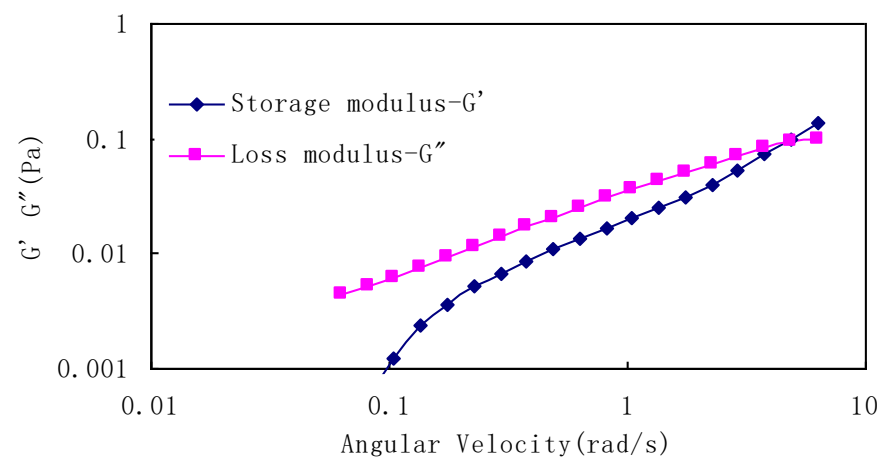

Figure 8. Storage Modulus and Loss Modulus Curve of the Polymer Solution

Polymer flooding stage: the recovery rate of the $189 \times 10^{-3} \mu^{2}$ permeability core is $6.2 \%$; the recovery rate of the $741 \times 10^{-3} \mu \mathrm{m}^{2}$ permeability core is $7.9 \%$; the recovery rate of the $21417 \times 10^{-3} \mu \mathrm{m}^{2}$ permeability core is $9.8 \%$.

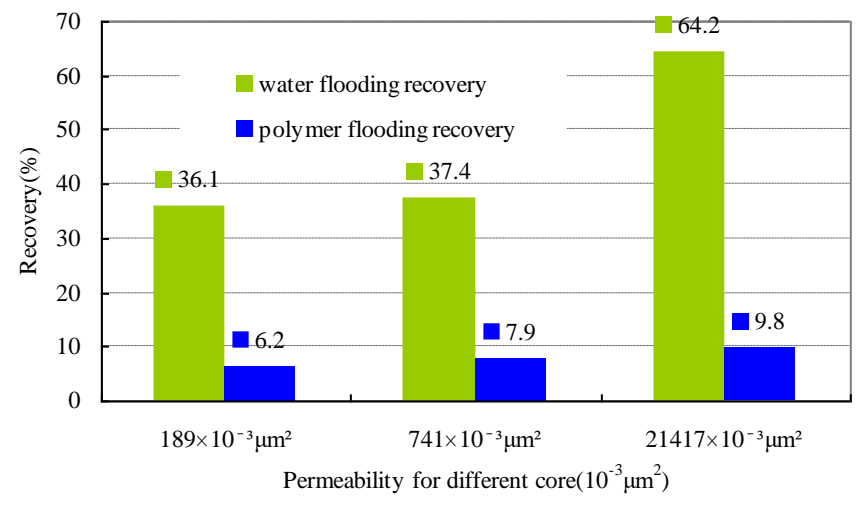

Figure 9. The Recovery Rate of Water Flooding and Polymer Flooding Stage 
The experiment result shows that: the recovery rate increases with the increase of the average pore radius and throat radius, and this trend is more obvious in water displacement experiment. In the polymer concentration under the condition of $1.0 \mathrm{~g} / \mathrm{L}$, the recovery rate also increases with the increase of the average pore radius and throat radius, but the trend is not obvious. In fact, oil saturation of the core with bigger pore and throat radius is already much lower than the other two cores in the water flooding stage. But the polymer flooding recovery rate is still higher than the two low permeability cores. So, relatively speaking, the increased recovery rate of high permeability core is higher than that of low permeability cores. The process of crude oil displacement in porous media is actually a process that the displacing fluid overcomes the capillary force. The bigger pore radius increases, the more capillary force decreases, and the more easily for the displacing fluid to overcome the capillary force. Thus there shows the phenomenon that the recovery rate increases along with the pore and throat radius.

\section{Conclusion}

In the penetration of the scope of the study (permeability: $189 \times 10^{-3} \mu \mathrm{m}^{2} \sim 21417$ $\times 10^{-3} \mu \mathrm{m}^{2}$ ), pore and throat work together to control seepage ability. With the increase of permeability, pore radius increases and the distribution range becomes wider; pore radius also increases, but the increasing extent is less than the increase in the throat radius. Pore structure of low permeability core is more complicated, so the distribution of pore-throat ratio is wide. High permeability cores of $21417 \times 10^{-3} \mu \mathrm{m}^{2}$, the number of effective pores of the analysis area is little because of the large cemented sandstone particle and pore size.

The pore and throat radius influence the effect of water and polymer flooding together. With the increase of pore radius and throat radius, the recovery rate of water flooding stage increases from $36.1 \%$ to $64.2 \%$; the recovery rate of polymer flooding stage increases from $6.2 \%$ to $9.8 \%$. The water flooding and polymer flooding recovery rate increases with the average pore and throat radius, and there is a trend that the increment increases along with the value of the average pore radius and throat radius.

This study only investigates effect of pore structure parameters of polymer flooding at the molecular weight in the same conditions. In fact, in order to achieve the ideal effect of polymer flooding, there should be one best matching relationship between reservoir pore structure and molecular weight of polymer. Therefore, the next we will continue to study on how the matching relationship of the polymer and pore volume distribution influence the effect of polymer flooding recovery.

\section{Acknowledgment}

This research was financially supported by The Youth Science Foundation of Northeast Petroleum University (project number:2013NQ122), National Science and Technology Major Projects (Grant No. 2011ZX05009-004, 2016ZX05010002-004 and 2016ZX05009003-010), Key Laboratory of Enhancing Oil and Gas Recovery of Education Ministry(Northeast Petroleum University) open subject: Non-destructive Testing Of Daqing Oilfield Class II Reservoirs Pore Structure Parameters. 


\section{References}

[1] Y. Zhou, J. Wang and Y. Yuan, "The Study of Polymer Flooding Mechanism and Influencing Factors", Science and Technology Innovation Herald, vol.12, (2011).

[2] Y. Zhu and Q. Hou, "Current Development and Application of Chemical Combination Flooding Technique", Petroleum Exploration and Development Online, vol.1, (2013).

[3] S. Iglauer, A. Paluszny and M. J. Blunt, "Simultaneous Oil Recovery and Residual Gas Storage: A Pore-level Analysis Using in Situ X-ray Micro-tomography”, Fuel, (2013).

[4] W. Ma and H. Xia, "The Effect of Rheological Properties of Alkali-Surfactant-Polymer solution on Residual Oil Recovery Rate after Water Flooding”, SPE, Brisbane, Australia, (2010).

[5] Y. Chen, J. Ni, J. Lihao, M. Liu, P. Wang and A. Rafig, "Experimental study on mechanical properties of granite after freeze-thaw cycling", Environmental Earth Sciences, vol.8, (2014).

[6] J. Wang and L. Liu, "A Novel Model and Sensitivity Analysis for Viscoelastic Polymer Flooding in Offshore Oilfield", Journal of Industrial and Engineering Chemistry, (2013).

[7] J. Gao, D. Han, J. Wang, "Research Oil Saturation Distribution in Core Water Flooding Using CT Imaging Technology", Xinjiang petroleum geology, vol.30, no.2, (2009), pp.269-271.

[8] H. Xia and D. Wang, "Viscoelastic Polymer Solution Experiment", Daqing Institute of Petroleum, vol.26, no.2, (2002), pp.105-108.

[9] P.Z. Wong, J. Koplik and Tomanic, "Conductivity and Permeability of Rocks", Physical Review B", vol.30, no.11, (1984), pp.6606-6614.

[10] S. Rodriguez, C. Romero and M. L. Sargenti, "Flow of Polymer Solutions through Porous Media", Journal of Non-Newtonian Fluid Mechanics, vol.49, (1993), pp.63-85.

\section{Authors}

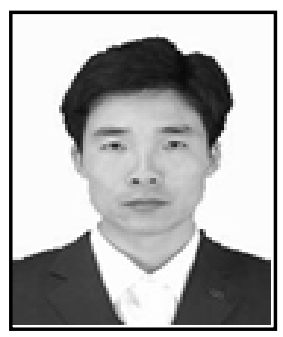

Wenguo Ma, he is an associate professor, master's tutor. Dr. Ma gets a PhD in Oil \& Gas Engineering from Northeast Petroleum University, Daqing, P. R. China. His recently research works mainly engaged in oilfield development engineering, oil production engineering and EOR technology, the main research contents include: the rheological property and the flooding mechanism of polymer solution and ASP system, chemical flooding microscopic displacement mechanism and the effect of reservoir pore structure on residual oil after chemical flooding by micro-CT.

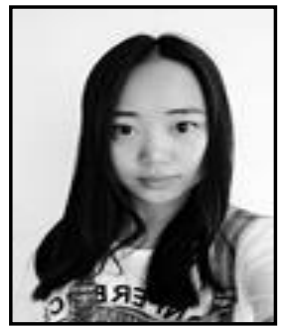

He Gong, she is studying at Northeast Petroleum University for a master's degree in oil and gas field development. Her main research direction is rheology of polymer solution, and enhanced oil recovery. 
International Journal of Control and Automation

Vol. 9, No. 10 (2016) 